\title{
Publication-Bias bei Psychotherapiestudien
}

Fragestellung: Führt ein Publication Bias, also die bevorzugte Publikation von Studien, die positive Ergebnisse zeigen beziehungsweise die Nichtveröffentlichung von Studien mit keiner Überlegenheit zu einer Vergleichstherapie, zu einer Überschätzung der Wirksamkeit von Psychotherapie bei depressiven Störungen?

Hintergrund: Eine frühere Studie derselben Arbeitsgruppe hat gezeigt, dass die Effektivität der Pharmakotherapie bei der Behandlung depressiver Störungen durch den Publication Bias überschätzt wird [1]. Mit anderen Worten reduzierten sich die Effektstärken um etwa ein Drittel, wenn in die metaanalytische Untersuchung der Wirksamkeit der Antidepressiva auch die nichtpublizierten Studien einbezogen wurden. Die Autoren untersuchten hier, ob ein Publication Bias auch einen Einfluss hat
Driessen E, Hollon SD, Bockting $\mathrm{CLH}$ et al. Does publication bias inflate the apparent efficacy of psychological treatment for major depressive disorder? A systematic review and metaanalysis of US National Institutes of Health-funded trials. PLOS One 2015; 10(9): e0137864 auf die Wirksamkeit von Psychotherapie bei depressiven Störungen.

Methodik: Im Gegensatz zu einer früheren Studie eines der Autoren [2], in der die Effekte eines Publication Bias auf die Wirksamkeit von Psychotherapie bei Depression durch statistische Modelle abgeschätzt wurde, gingen die Autoren hier anders vor: Sie identifizierten alle durch das NIH in den USA finanzierten Psychotherapiestudien zur Depressionsbehandlung zwischen 1972 und 2008 und untersuchten, ob diese zu Publikationen geführt hatten.

Falls dies nicht der Fall war, kontaktierten sie die Autoren und baten um Bereitstellung der Daten. Daraus kalkulierten sie die Effektstärken für die nicht-publizierten und die publizierten Studien und untersuchten, wie stark sich die Effektstärken für Psychotherapie veränderten, wenn alle Studien zusammen betrachtet wurden.

Ergebnisse: Von den 57 finanzierten Studien wurden 13 (23,6\%) niemals publiziert und zwei Studien nicht begonnen. Die 13 unpublizierten Studien wiesen eine Effektstärke gegenüber Kontrollbehandlungen von 0,20 auf $(95 \% \mathrm{KI}-0,11$ bis 0,51 ; n. s.) im Gegensatz zu den publizierten Studien mit einer Effektstärke von 0,52 (95\% KI 0,37 bis 0,68). Wenn man alle Studien zusammen betrachtete, reduzierte sich die Effektstärke für Psychotherapie-Effekte von 0,52 um $25 \%$ auf 0,39 (95\% KI 0,08 bis 0,70 ), was immer noch signifikant war.

Schlussfolgerungen: Psychotherapie bei depressiven Erkrankungen ist wirksam, aber nicht in der Stärke, wie es ohne Berücksichtigung des Publication Bias behauptet wird.

\section{- Kommentar von Klaus Lieb, Mainz}

\section{Verzerrungsrisiko nicht nur bei Pharmastudien}

Die Studie ist wichtig, da sie zeigt, dass Verzerrungsrisiken bei der Beurteilung von wirksamen Therapien nicht nur bei Pharmastudien auftreten, sondern eben auch bei Psychotherapie. Die hier vorgelegte Studie zeigt das anhand einer relativ kleinen Zahl von Psychotherapiestudien, die vom NIH in den USA finanziert wurden. Vergleicht man die Ergebnisse mit den früheren Analysen derselben Arbeitsgruppe [1] zeigt sich, dass die Effektstärken für Antidepressiva infolge des Publication Bias um einen ähnlichen Prozentsatz (zirka $33 \%$ ) sanken wie bei den hier untersuchten Psychotherapiestudien. Die Daten sind auch von der Größenordnung vergleichbar mit der Studie von Cuijpers aus dem Jahr 2010, der eine Reduktion der Effektstärken von Psychotherapie bei Depression von 0,67 auf 0,42 bei Berücksichtigung des Publication Bias durch statistische Modelle abgeschätzt hatte [2].

Fazit: Erstens unterstreichen die Ergebnisse, wie wichtig es ist, dass alle Studien registriert und deren Ergebnisse zumindest in öffentlich zugänglichen Registern publiziert werden, damit die echten Therapieeffekte ermittelt werden können. Zweitens sollten die Ergebnisse zu mehr Besonnenheit bei denjenigen beitragen, die Medikamente zur Behandlung von Depressionen zugunsten von Psychotherapie kritisieren. Die Effektstärken von Psychotherapie bei Depressionen liegen um die 0,4 und sind damit klein bis mittelgroß und überschreiten nicht die Schwelle von 0,5, die von vielen Autoren als klinisch relevante Schwelle angesehen wird. Drittens sollte eine verstärkte Debatte geführt werden, ob unser auf positive Befunde ausgelegtes Publikationswesen (es ist heute sehr schwer, negative Studienergebnisse zu publizieren!) noch zeitgemäß ist.
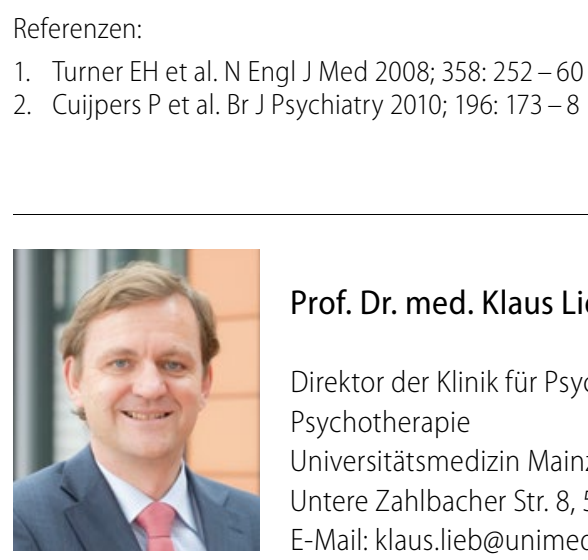

Prof. Dr. med. Klaus Lieb, Mainz

Direktor der Klinik für Psychiatrie und

Psychotherapie

Universitätsmedizin Mainz

Untere Zahlbacher Str. 8, 55131 Mainz

E-Mail: klaus.lieb@unimedizin-mainz.de 